\title{
A Study on Pyramid Scams in China and Its Precautions
}

\author{
Te Zhang \\ Zhongnan University of Economics and Law \\ Wuhan, China
}

\begin{abstract}
As the communications between China and the rest of the world getting more and more frequent, China's public security has impressed a lot of foreign visitors. However, when it comes to pyramid scam, it's a completely different story: today, there is hardly a place anywhere else with a similar situation where pyramid scam can build up such a scale, and can be this hard to eradicate. In this paper, the writer will take a closer look on several distinct pyramid scam cases happened in China, trying to understand it, and figure out some ways to counter it.
\end{abstract}

Keywords—pyramid scams; precautions; illegal

\section{INTRODUCTION}

Among all versions of rumor regarding how exactly pyramid sales was introduced into China, the most tenable one is the HTE international Group ${ }^{1}$ from Taiwan has first done so in 1992, its Chinese mainland branch was "Xingtian Company". The product they were selling is a piece of health care device called "Shuang-Ankang aerobic fitness oscillator" 2 . The product costs $400 \mathrm{CNY}$ to manufacture, and the company set the price to more than $6000 \mathrm{CNY}$. The company adopted a member managing system called "five level-three order system", meaning there are five levels in total, each consists of three orders. Members can climb up the ladder order by order, as long as they keep completing the required sales task designed by the company, whoever climbed to the highest order, are promised to be rewarded with a tremendous amount of money. Notice all the sales personnel are not really employees, for there isn't a valid contract of service in existence. Salesmen join the business by purchasing the product and paying an entrance fee for "membership" spontaneously, the "membership" means you're in the "five level-three order system", and can cut a share every time you've introduced a new member. The business has grown so influential that a small agency of the company held a conference in Changsha, Hunan province, appealed tens of thousands of people trying to get into the conference hall. Some attenders were too desperate trying to

Written as 兴田企业股份有限公司 in Chinese, for reference. Written as 爽安康有氧健康摇摆机 in Chinese, for reference. get in, the conference soon turned into a large scale fist fight and was forced to cancel. ${ }^{3}$

As the business growing, the production of their machine can no longer keep up with the demand, and the actual effect of the product is never put into consideration by anyone involved. At this point, the business has transformed from selling real products to purely recruiting new members for their entrance fee, and then returned them to older members as revenue, a pyramid scam. By that time, Chinese government has just finished the reformation from planned economy to market economy, the inexperience resulted in a rather slow response time to the pyramid sales, and multiple severe social incidents erupted. Official response finally came out in April 24th, 1998, declaring all kinds of pyramid sales as illegal activities.

\section{GENERAL Picture OF ILlEGAL PyRAMID SALES IN CHINA NOWADAYS}

After the nation-wide banning, "Xingtian Company" was mobbed by a mass crowds demanding refund, the company had to declare bankrupt due to the inability to fulfill its liability of refund. However, quite a number of the key members in the company managed to avoid being called to account, and kept on their pyramid sales career. The remaining pyramid selling network left by the formal "Xingtian Company" was still partially active and later divided into two factions: Northern and Southern, these two factions created the situation of illegal pyramid selling crimes in China nowadays.

Although named geographically, the two factions are actually distinguished by operation pattern.

The Northern faction is derived from a former agency of "Xingtian Company" in Wuhan, Hubei province. The key members there left to the North-Eastern area of China (Heilongjiang, Jilin and Liaoning province), to start pyramid scam. Its influence has disseminated into Hebei, Tianjin and Shandong province in 1997, then to all other areas in China. The pattern is to release fake information about employment or training to the society to lure the victims to rural areas, then suppress the resistance of the victim with deception or

\footnotetext{
3 Tencent: Pyramid selling's 28 years in China: Originated from Japan, derived into two fractions. http://tech.qq.com/a/20170807/024216.htm
} 
violence under the cover of so called "will power training". The victims will get minimum standard accommodation just enough to be kept alive, usually eating leftovers collected from nearby markets by themselves. The freedom of person and communication are prohibited. All these activities is to keep the victims imprisoned with a very low cost, so the organizer can then carry out a series of carefully designed collective propaganda, preaching about "interpersonal relation marketing", "factory outlet" in their own twisted way, using a highly overpriced or a non-existing "product" to cover up the iconic characteristic of pyramid scam, which is using the "entrance fee" from the new members as the revenue for the old members. In the process, whoever tries to escape or discovers the deception and try to debate (especially under the witness of other victims) outside the designed propaganda, will be threatened, beaten or even murdered by the organizers of the scam. After the propaganda, most of the victims are brainwashed and then granted limited access to cellphone and internet to contact their relatives and friends to join the scam, some are threatened, and some are deceived.

The majority of the victims of Northern faction pyramid scam are youngsters aged around 20. Victims also include other mid-low income personnel seeking better working opportunities, usually less educated.

The Southern faction appeared rather late. Led by the former key members of "Xingtian Company" who managed to dodge the punishment of the government, in comparison with the Northern faction, the Southern faction focuses more on delicate deception strategy. Southern faction usually uses fancy concepts like "chain sales", "capital operation", "nongovernmental mutual aid financing" etc., to blend their scam in. They prefer taking advantages of aged people's blind worship toward government derived from the time when China was adopting planned economy system. A conventional trick of the Southern faction is to distort or make up speeches of important politician, sometimes even forge documents issued by the government. Their method varies, but the idea is nothing more but to mislead people into believing the scam is supported by the government. Other than taking advantages of people's trust on the government, Southern faction scam organizations usually have a set of carefully designed plea, to blur the boundaries between their scam and legit businesses; some even own printing presses to mass spread their "theory", tricking random people joining the scam. By faking the support of the government, and the legality of the "business", what victims see is a golden chance for investment, legal and without any chance of deficit. What's similar to the Northern faction is that scam patterns of Southern faction also include luring victim to a place he or she isn't familiar with, but under the cover of "visitation of the project", or simply a "PR tour for potential shareholder/client". The organizers of the scam will talk nicely to each victim individually during the victim's stay at hotel, pretend trying to be friends with the victim, and convince the victim to join the pyramid scam, the victim is free to leave in this pattern, the aim for taking victims to a different place, is to block the possible interference from the victims' friends and relatives, some scam involves showing current "progress of the project", increasing the persuasiveness of the scam. ${ }^{4}$

Although there are also youngsters or mid-low workers looking for a better job in the Southern faction scam, their prioritized targets are people with quite some money at disposal. The high resemblance to ordinary business of this kind of scam, plus the carefully designed traps, makes the deception very hard to detect even with a high level of knowledge. One typical case is the "1040 Sunshine Project", the defrauded amount went more than 600 million Chinese Yuan. The case contains another scam disguised as bitcoin investment. Although key members are arrested and sentenced to imprisonment, the scam organization is still running, with former underlings taken the place of their old leaders, they're still tricking people and didn't even change their "brand". But instead of one concentrated large group, they're now operating in small groups spreading all over the nation, making them very hard to eradicate by the government as a whole.

In general, the two factions operate in different ways, but the essence is shared: remote solicitation, charging an entrance fee, pulling more people joining the scam. As time goes by, the two factions are drawing lessons from each other, the pattern of their crime activities are evolving. At present, several tendencies can be observed.

First, blurred lines between business and crime. With the accelerating development of Chinese economy, new business models are invented, and we haven't figured out what to do with them. For instance, in August, 2011, Jiangxi province, a case known as "Pacific Direct Buying case". In this case, there was a website called Pacific Direct Buying, its behavior is rather similar to "Massdrop", which uses internet to gather a large group of people with the same product they wish to buy, then the website will contact the manufacturer directly, in order to get a lower price and more complimentary gift than normal retail sources. What makes the website suspicious is that the website will ask for a certain amount of deposit as guarantee, in case the buyer canceled order during the negotiation between the website and the manufacturer. What's more, the website came up with an idea of allowing members to trade the gift from the manufacturer, before the gift is sent by the manufacturer, and whoever introduced another user to the website will get a significant bonus on their credibility score. Higher the score is, the more rebate the user can get from each sale. These actions have drawn the attention of the procuratorate. Because the credibility score system combined with deposit of guarantee, does sound like pyramid scam indeed, especially the things they

\footnotetext{
4 Tencent: Violent Northern, Gentle Southern, How did pyramid scam sweep across China.

http://view.news.qq.com/original/intouchtoday/n3973.html

Chinese anti-scam web: "1040 Sunshine Project" Pyramid Scam in Beihai, Guangxi province has tricked too many people. http://www.fcxzyz.com/nanpai/ziben/2017-04-07/3393.html

Written as 太平洋直购案 in Chinese, for reference.
} 
were selling aren't even real. The case closed with a "guilty as charged", but the arguments are far from settled. ${ }^{7}$

Second, the changes of members' subjective aspects. In conventional pyramid selling cases, new members of the scam organizations are mostly tricked or forced into the scam, which means these new members don't know what they've been doing, thus they are innocent. However, some new groups have applied a different strategy. They've introduced "greater fool theory" into their persuasion, they admit it's a scam out right, and then try to persuade the new members by telling them there is always a fool willing to pay for the myth and buy the useless and overpriced "product" in the sam, joining the scam is a fast way making money. In this scenario, the new member joined the scam with an intention of fraud, these types of personnel can no longer be called "victims" they're genuine perpetrators. In Chinese criminal law, only the key members of the pyramid scam are considered criminals, when someone joined the scam with an intention of fraud, can that person still be considered innocent? If so, what is the reason for excuse? If not, how do we integrate the crime of fraud with illegal pyramid selling? These are the questions yet to be answered.

\section{A CLOSER LOOK ON THE WIDELY USED METHODS IN PYRAMID SCAM}

\section{A. “Brain Wash” Methods}

According to the testament of escaped victims, and settled cases in the past, the writer of this paper has summarized four tricks commonly used by pyramid scam organizations.

1) Creating a false image of having a governmental background: Take the nation-shocking "Maya pyramid scam" ${ }^{8}$ as an example, In early 2005, A company called Maya Biotechnology was confirmed to have conducted illegal pyramid selling, 200 million Chinese Yuan was involved in the case. The company hired several research facilities to issue fake experiment report showing the product is useful and of high technology, and the company claimed itself to be "subordinate to the National Technology Development Committee of Nanning Province", the company has a legal registration of incorporation, all permission was acquired legitimately. To deceive people further, the company deliberately chosen to broadcast its advertisement right after the end of widely trusted television programs about consumer rights, like " 315 " and Billboard of Integrity“9 . The company also brags about the promising future of the company through newspapers and its own publications. These eye-dazzling propaganda has led lots of

Qing Zhu. Pyramid scam or innovation: A Legal analysis of the "Pacific direct buying" case[J]. Studies in Law and Business, 2015,32(01):71-80.

Sina: "Maya" Pyramid scam from start to end: 200 millions of entrance fee for a myth getting rich overnight.

http://news.sina.com.cn/c/2006-03-17/07468461199s.shtml

9 Written as 诚信金榜 in Chinese, for reference. people into the trap, and firmly believed they're on the Fast track to wealth.

2) Making the entrance fee looks negligible: In the "Maya" case mention above, another phenomenon is shared with many other cases, that's all of them are trying to make the entrance fee look negligible by promising a huge revenue. The entrance fee charged by "Maya company" is $288 \mathrm{CNY}$, and the company gives back so called "144 CNY worth of product" which actually worth nothing, making the entrance fee looks even cheaper. According to the company, a mere 288 Yuan of investment, can have 1060 Yuan in return after 6 months, and there is a 19988 Yuan bonus after 17 months. This particular scam is using the greed of the victim, because once someone believed 288 Yuan can generate more than 20 thousand revenue, they'll invest as much as possible rather than just pay 288 Yuan. Another example is the also well-known "1040 Sunshine Project" case, claiming anyone bought a share with 69800 Yuan, and introduce 29 other "shareholders", can have 1040 "wan"10 Yuan as revenue, hence the name.

With such a high revenue making the "business" tempting, and a detailed calculation making it looks promising, even if the victims have doubt on the "business", it'll be very hard for them to simply walk away, because it can be true and the revenue is just too much to resist. Lots of victims joined the scam thinking "The entrance fee is not much, it won't hurt even if it's a trap, but it's really a shame if it's real." But gradually, they'll always get greedy under the repeated persuasion of the organization, and handed over all their savings.

3) Disguised replacement of concept: "versatile chain sales" and "product selling": When questioned about if it's a pyramid scam, the scam organizer will always try mixing pyramid scam with normal chain sales pattern. They keep emphasizing on pattern of their operation, repeating chain sales pattern is keep searching for lower tier distributer, while paying to higher tier supplier, which is essentially the same as a pyramid scam, just more versatile. What they won't talk about, is the "product" in pyramid scam worth nothing compared with its designated price, there is no actual demand. While in normal chain sales, the price is much closer to the merchandise's real value. This decisive part of difference is deliberately neglected.

4) Using the inclined "polarizing effect" to urge victims to join: Psychologists noticed that decisions made by a group of people tend to be more bold and risky than normal, they called this phenomenon "risk shift". But after further research, they realized decisions made by a group can also tend to be more conservative than normal too, depending on the exact case. And on the aspect of individuals, personal attitude toward risk is amplified when making group

\footnotetext{
10 “wan" (万) is a digit position in Chinese, 1 wan=10 thousand The reason writer didn't use thousand, is to show the logical relationship between 1040 wan and the "brand" of the scam.
} 
decisions. Moscovici \& Zavalloni (1969) called this phenomenon "group polarization". 11

It doesn't matter if the designers of the scam actually know this theory, because they're obviously putting it to use. For most of the pyramid scam groups, they all adopt group discussion after the propaganda "lecture", to let their victims think about what has been said. Of course it's not really free discussion, there's always some already brainwashed old members mixed in the group, disguised as well-educated new members. During the discussion, these old members are going to act like a catalyst, because they'll behave very stimulated and engaged on their "new career", their influence will have a significant impact on those who're still indecisive, and veer the majority of the group into believing the scam, and those who still have doubts will also choose to believe in order to fit in. When tendency is ensured, the discussion of the whole group becomes a process of polarizing, leading people in to believing the scam more and more.

\section{B. Analysis on the Cause of the Crime}

1) Anomie theory: According to anomie theory, it is the social norm that holds the order and culture of the society in place, which provides interactions of the society a context. It's safe to say social norm is guiding the motion of the society. Once a new social norm risen, there'll always be a time when the old norm can no longer accommodate the need of the society, while the new social norm isn't strong and sophisticate enough yet, to hold the society together. During this period, social norm can't keep social activities within its bounds, this is state is what we call "anomie". The word "anomie" is first used by French sociologist Emile Durkheim (1858-1917), when talking about the various circumstances of social participation. Durkheim believes, self-realization and acquisition of happiness can only be possible when all social norms can form a complete system without conflict in itself. Under this basis, an individual can interact with others on a moral level, and happiness can be achieved in a clear boundary, all these will never happen when the society is in anomie.

This theory can be persuasive when we're trying to understand the reason of China's pyramid scam. In fact, ever since the "reform and opening up" policy, the huge Chinese economy growth changed everything. The traditional Chinese moral standard mixed with new social norm imported from abroad, the contradicting social norms caused disorder in every aspects. This is the "anomie" mentioned by Durkheim: Old social norm is failing while a new one isn't fully acknowledged yet, people don't know what to believe, and this is where pyramid scams comes in, simply depict an insane fantasy and people will believe it, because everything is undefined. ${ }^{12}$ According to the testimony of the victims can be found online, these victims wouldn't pay effort, wouldn't

11 Yanping Gong, Sheng Chen. Literature review of studies on group polarizing phenomenon. Value Engineering, 2013,32(32):149-150.

Yuzhen Xia. Anomie and regulation of Chinese society during transition period[J]. Journal of Central China Normal

University(Humanities and Social Sciences), 2002(05):28-32. take any risk, and they believed it's that easy being overnight billionaires; They want to be proven capable and resourceful by doing this "business", but their choices are dependent on their imaginary "national support". These double-standard logics clearly show they don't have a standard in their mind.

2) Strain theory: The concept of "anomie" is widely accepted after Durkheim, but its meaning varies. American sociologist Robert King Merton (1910-2003) deems "anomie" as a state of the society that the universally acknowledged goal (cultural goal) is incompatible with the norms (institutionalized means) when reaching for the goal. ${ }^{13}$ Cultural goals and institutionalized means are the two related key factors in his theory. According to Merton, when people can't achieve the cultural goal by institutionalized means, especially others have successfully achieved the goal; people would experience frustration or anger. To ease the stress of being left behind, five attitudes can be seen. But in terms of means, crime or deviance are usually the answers. ${ }^{14}$

As mentioned above, when everything is undefined, people don't know what to believe, but one thing is certain: whoever lives better is closer to success. So people undesignedly agreed being rich is success, become rich is then set as a cultural goal. Later, people found out this cultural goal are irrelevant with institutionalized means like study or work hard. In contrary, it's usually those who dropped out of high school became the rich business owners, that's when people start to look for new ways getting rich, again, that's where pyramid scam comes in. The initiators and organizers are basically normal crime against property, nothing special, but what are interesting is the victims that got involved. The writer thinks they can be considered as "innovation" by Merton's standard, they agree on the cultural goal (getting rich), but seek new means of reaching the goal, despite being tricked into tricking others.

\section{PReCAUtions to Be TAKen By Chinese GOVERNMENT}

\section{A. Improving Crackdown Strategies}

The aftermath of "Xingtian Company" and the still ongoing "1040 Sunshine Project" pyramid scam are teaching us an important lesson: It's not going to work if we indiscriminately imitate existing experiences on fighting other crimes with organization.

In the writer's opinion, there are two fundamental differences between pyramid scam and other crimes with organization. As the typical organized crime pattern is underworld gangs, we'll take that for an example. Normally, when we're dealing with an underworld gang, we can use brute force to crush their resistance head on, because all of the gang members are fully aware of their actions and

13 Zhaoning Yang. Two theoretical commentary on Individual facing strained circumstances[J]. Psychological Exploration, 1999(03):5358.

14 Xinghua Guo. Social anomie and devience[J].Journal of Huaiyin Teachers College(Social Sciences Edition), 2002(01):35-38. 
consequences, therefore they can be fought as criminals. However, in pyramid scams, although they also need an organization, members of a pyramid scam organization usually don't understand what the real situation is, hence most of them are considered innocent victims. This fact significantly limited our methods when resolving the case. Also, the existence of a gang relies on financial support that needs key members to operate. Capturing the key members of a gang means cutting its source of income, this will cause mass destruction on the gang's organization network, and most of it will dissipate on their own. But the income of a pyramid scam organization is from its tricked new members, capturing key members of a pyramid scam organization won't damage the organization at all, the former underlings can easily take the place, and the organization can keep growing.

Our conventional method of dealing with pyramid scams is to punish initiators and the organizers of the scam only. But early members of the organization who didn't quit, also seen how their "business" runs, they have far more opportunities to realize it's a scam but they choose to follow. When these people claim to be innocent, the writer doesn't think they should be spared.

Also, to cut the source of the scam organization, we'll have to rely on people's ability to detect a scam themselves; we've put a lot of effort on propaganda, trying to prevent people from believing the scams, which is great. But we need to put more consideration into what to do with the already caught participants of pyramid scams. When a pyramid scam organization is demolished, the key members are sent to the court, but its brain washed members aren't paid enough attention to. Many of them still believe in what they've been told, and will be joining another pyramid scam as soon as they're released, some may even start a new one. ${ }^{15}$ These people might be innocent, but there is no doubt they can be dangerous to the society. Hence we do need to limit their personal freedom temporarily, make sure they understand the so called "business" is merely a myth, before letting them get back to society. This limitation of freedom is not punishment, but a crime prevention measure.

\section{B. Creating an Active and Dynamic Society Environment}

As chairman Xi Jinping has already mentioned during the 19th NPC (National People's Congress), we've now fundamentally ensured the material well-being of the people, our next mission is to build a co-construction, multigovernance, wide-sharing society in China.

As of now, regardless of urban-rural, interregional or inter-stratum level, the fruit of Chinese development is distributed in an imbalanced way. With the view of the anomie theory, the reason of vast spread of pyramid sales scam can be charged upon the huge economic achievement of the society, which has caused the disjoint of social norm and the reality. And according to strain theory, people are

15 Yanan Zheng, Wen Hu. A mental intervention case on a pyramid scam victim [J]. Medicine \& Philosophy (B), 2017, 38(12):70-73. Yao Chenggao, Huang Renzhi. Psychoanalysis and rectify of Pyramid scam victims [J]. Journal of Sichuan Police College, 2006(01):59-62. easily lured into the pyramid scam because of the imbalanced distribution of our achievements, which is confusing the public on whether we should be sticking the conventional value standard or not.

What's more, the vast spread of pyramid scam also reflects our financing channel and entrepreneurial environment are yet to be improved. When paying for the pyramid sales myth, lots of victims are motivated by the expectation of earning revenue in the future. To a certain extent, this means the society's absorptive capacity toward cash is insufficient, to fight illegal pyramid sales, we should also take peoples' need for investment into consideration, government should guide funds into the areas that actually can drive the society forward, while educating people with a formidable, scientific theory. Only in this way can we truly have a chance eradicating the pyramid scam.

In terms of victims, college students became a constituent part. In the eyes of the writer, this, on one hand, shows there aren't enough jobs for these students in the society. On the other hand, shows the desperate desire of these youngsters to get on the track of their life. Thus, the topic of constructing a fair and transparent entrepreneurial environment has become the elephant in the room, again. There is no doubt, with an improved financing channel, these youngsters can create a future we could never have dreamed of.

\section{CONCLUSION}

For decades, China has been focusing on elevating peoples' material living standard, the effort is proven to be fruitful. Life without famine was once our dream, and now we're enjoying high quality infrastructures the world envies, the time has changed a lot, indeed. But new problems rises with our steps forward, China's advancement is now meeting a bottleneck, we're facing the painful and risky structural transformation on the scale of entire society if we're to progress further, promoting material civilization along can no longer satisfy our need, we also need to improve our social system, making the society an everlasting mechanism that can drive its people to a brighter future. The writer deeply hopes introspections in this paper can arouse more attention to system construction.

\section{REFERENCES}

[1] Mingkai Zhang. Basic matters of pyramid selling crime[J]. Political Science and Law, 2009(09):27-33.

[2] Xingliang Chen. Crime of organizing, leading pyramid scam activities: Nature and Boundries[J]. Tribune of Political Science and Law, 2016,34(02):106-120.

[3] Yanan Zheng, Wen Hu. A mental intervention case on a pyramid scam victim[J].Medicine \& Philosophy(B), 2017,38(12):70-73.

[4] Likun Lv. An analysis of juvenile delinquency with a view of strain theory[J]. Legality Vision, 2017(12):219.

[5] Qing Zhu. Pyramid scam or innovation: A Legal analysis of the "Pacific direct buying" case[J]. Studies in Law and Business, 2015,32(01):71-80

[6] Xiaoxi Shi. New characteristics and cause of Pyramid scam at present and its prevention. Legal and Economy, 2014(03):48-49. 
[7] Yanping Gong, Sheng Chen. Literature review of studies on group polarizing phenomenon. Value Engineering, 2013,32(32):149-150.

[8] Chenggao Yao, Renzhi Huang. Psychoanalysis and rectify of Pyramid scam victims[J]. Journal of Sichuan Police College, 2006(01):59-62.

[9] Yuzhen Xia. Anomie and regulation of Chinese society during transition period[J]. Journal of Central China Normal University(Humanities and Social Sciences), 2002(05):28-32.

[10] Xinghua Guo. Social anomie and devience[J].Journal of Huaiyin Teachers College(Social Sciences Edition), 2002(01):35-38.

[11] Zhaoning Yang. Two theoretical commentary on Individual facing strained circumstances[J]. Psychological Exploration, 1999(03):53-58.

[12] Sohu: Chinese pyramid scam distribution map. https://www.sohu.com/a/162491521_208178

[13] Tencent: Violent Northen, Gentle Southern, How did pyramid scam sweep http://view.news.qq.com/original/intouchtoday/n3973.html

[14] Tencent: Pyramid selling's 28 years in China: Originated from Japan, derived into two fractions. http://tech.qq.com/a/20170807/024216.htm

[15] Baidu: Pyramid scam distribution map of China has come out, why is Guangxi province the No.1 disaster area? http://baijiahao.baidu.com/s?id=1565073015485982\&wfr=spider\&for $=\mathrm{pc}$

[16] Chinese anti-scam web: "1040 Sunshine Project" Pyramid Scam in Beihai, Guangxi province has tricked too many people. http://www.fcxzyz.com/nanpai/ziben/2017-04-07/3393.html

[17] Sina: "Maya" Pyramid scam from start to end: 200 millions of entrance fee for a myth getting rich overnight. http://news.sina.com.cn/c/2006-03-17/07468461199s.shtml

[18] Zhihu Community: How powerful is the brainwash of pyramid scam? https://www.zhihu.com/question/35776863/answer/136887483

[19] Sohu: Reports claiming the government is conniving small pyramid scams. http://www.sohu.com/a/106282559_162903

[20] Baidu: Pyramid scams have a surprisingly long history? https://zhidao.baidu.com/question/629559121334994484.html 